Int. J. Dev. Biol. 48: 1155-1158 (2004)

doi: $10.1387 / \mathrm{ijdb} .041917 \mathrm{ht}$

Developmental Expression Pattern

\title{
Identification and developmental expression of Xenopus paraxis
}

\author{
HSIU-TING TSENG ${ }^{1}$ and MILAN JAMRICH*,1,2,3 \\ ${ }^{1}$ Department of Molecular and Human Genetics, ${ }^{2}$ Department of Molecular and Cellular Biology and ${ }^{3}$ Program in Developmental Biology, \\ Baylor College of Medicine, Houston, USA
}

\begin{abstract}
During vertebrate embryogenesis, the paraxial mesoderm becomes segmented in a rostro-caudal progression and gives rise to the somites. In this paper we report the isolation of a Xenopus orthologue of paraxis, a member of a family of basic helix-loop-helix proteins, which has been suggested to play a role in paraxial mesoderm development. Xenopus paraxis is initially expressed in the presomitic paraxial mesoderm and later in the dorsal portion of the developing somites. Finally, paraxis expression becomes restricted to the most dorso-lateral region of mature somites.
\end{abstract}

KEY WORDS: paraxis, bHLH, somite, paraxial mesoderm, dermatome

The metameric organization of the vertebrate body is first revealed during embryogenesis by the segmentation of the presomitic mesoderm into somites. In all vertebrates, segmentation proceeds in a rostral-to-caudal wave and gives rise to somites which bilaterally flank the notochord and neural tube. Mature somites are differentiated into three compartments: dermatome, myotome and sclerotome, which form the dermis, trunk musculature and axial skeleton of the adult structures. Although the initial and completed states of somitogenesis are similar in all of the vertebrates, the morphogenetic processes vary from one species to another. In Xenopus, blocks of mediolaterally elongated cells form somites by simultaneously rotating 90 degrees to lie with their long axes parallel to the anterior-posterior axis (Hamilton, 1969; Youn and Malacinski, 1981). In addition, the small dermatomal and sclerotomal cell population are so inconspicuous that little is known about the patterning of the somites into dermatome, myotome and sclerotome (Keller, 2000). Therefore, one of the major challenges in this experimental system is to develop molecular markers for major components of the developing somite.

paraxis is a basic helix-loop-helix (bHLH) transcription factor expressed in paraxial mesoderm and later localized in the dermatome compartment of the somites, first cloned in mouse and chick (Burgess et al., 1995; Barnes et al., 1997). In the absence of paraxis function, the axial skeleton and skeletal muscles form, but are not correctly patterned (Burgess et al., 1996). paraxis is nearly identical to another bHLH gene, scleraxis (Cserjesi et al., 1995; Brown etal., 1999), within the bHLH region and shares $84 \%$ nucleotide identity in the entire coding region in mouse. As the somites compartmentalize in the developing mouse embryo, paraxis expression is maintained in the dermatome and sclerotome. At this stage, paraxisand scleraxisare co-expressed in the sclerotome, but paraxis expression declines after sclerotome formation whereas scleraxis expression increases (Burgess et al., 1995). Since paraxis and scleraxis are structurally highly related, we used the mouse scleraxis cDNA to screen for both genes in a Xenopus genomic library. This screening resulted in the isolation of the Xenopus orthologue of paraxis, which is the topic of this paper. We found no evidence that a scleraxis orthologue is present in the Xenopus genome.

\section{Results and Discussion}

\section{Identification of a Xenopus paraxis orthologue}

Screening a Xenopus genomic library with the mouse fulllength scleraxis led to isolation of five positive clones. Sequence analysis of these clones revealed that these clones encode the Xenopus orthologue of paraxis. Xenopus paraxis contains an open reading frame of 579 nucleotides encoding a protein predicted to be 193 amino acids.

Comparison of the deduced amino acid sequence of Xenopus paraxiswith chick (Barnes etal., 1997), zebrafish (Shanmugalingam and Wilson, 1998), mouse (Burgess et al., 1995) and human

Abbreviations used in this paper: bHLH, basic helix-loop-helix.

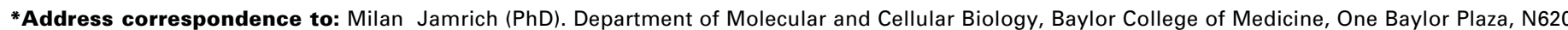
Houston, Texas 77030, USA. Fax: +1-713-798-3017. e-mail: jamrich@bcm.tmc.edu
} 
A

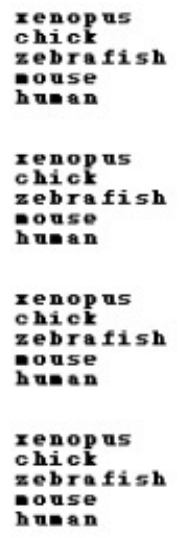

xenop

zebrafish

ebrafish

\section{5
6}

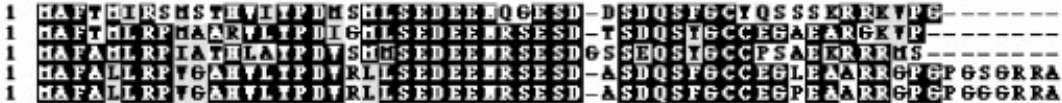

1 WAFALL RPFGATTIP DFRIL SEDEETR SE SD-ASDOSFGCCEGIEAARRGPGP GSGR RA
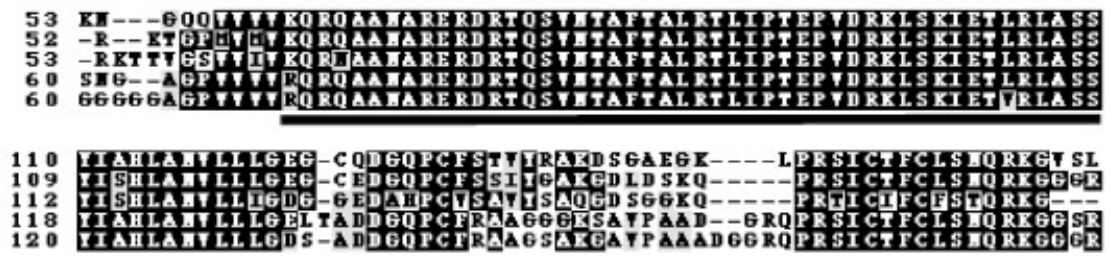

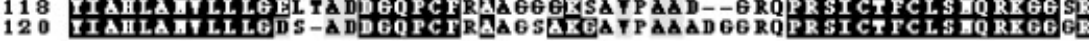

\section{AL GT SHCFAOSLGE RGHP QGAHTL GHRS}

163 EDLGGTCLETRGYTPLET ARP

163 IRDLTDCIRHRGIASL RHF SE

176 ROGESCIRTRG APL REPRR

B

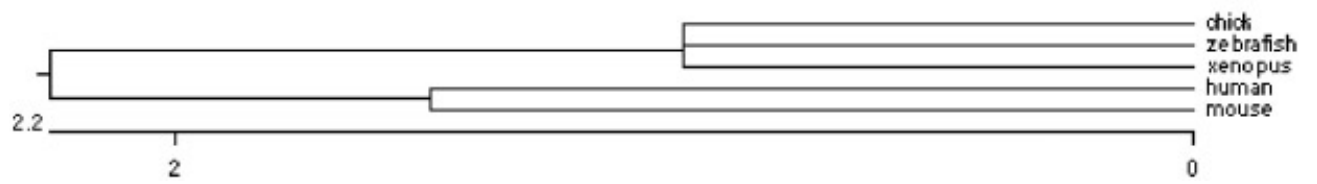

Fig. 1. Analysis of paraxis protein sequences. (A) Amino acid sequence comparison between Xenopus paraxis and the orthologues in other species. Identical amino acids are shaded black and conserved changes are shaded gray. The absence of residues at the corresponding region is indicated by dashes. The basic helix-loop-helix domain is underlined. (B) Phylogenetic tree for paraxis in Xenopus, zebrafish, chick, mouse and human prepared using the DNAstar program using the J. Hein method with PAM250 matrix (Hein, 1990). The scale shown is the divergence calculated in the distance matrix.
(Quertermous et al., 1994) Paraxis protein reveals 77, 69, 68, 66\% sequence identity, respectively (Fig. 1A). Alignment of all currently available Paraxis sequences from different species shows that the basic helix-loop-helix region is highly conserved and that the carboxy-terminus is most divergent across species (Fig. 1A). A phylogenetic tree based on amino acid comparisons within the bHLH region indicates that the Paraxis orthologue of chick, zebrafish and Xenopus form a group distinct from mammalian orthologues of Paraxis (Fig. 1B). In this subgroup, scleraxis was only found in chick (Schweitzer et al., 2001), implying a differential regulation of somite development between lower and higher vertebrates (Morin-Kensicki and Eisen, 1997; Keller, 2000).

\section{Temporal expression of Xenopus paraxis}

The temporal expression of paraxis was analyzed by RT-PCR using total RNAs isolated from different developmental stages (Fig. 2 ). The expression of paraxis begins during late gastrulation and continues throughout the tadpole stages. paraxis transcripts are most abundant during the period when the mesoderm forms somites in Xenopus development (stages 17-24).

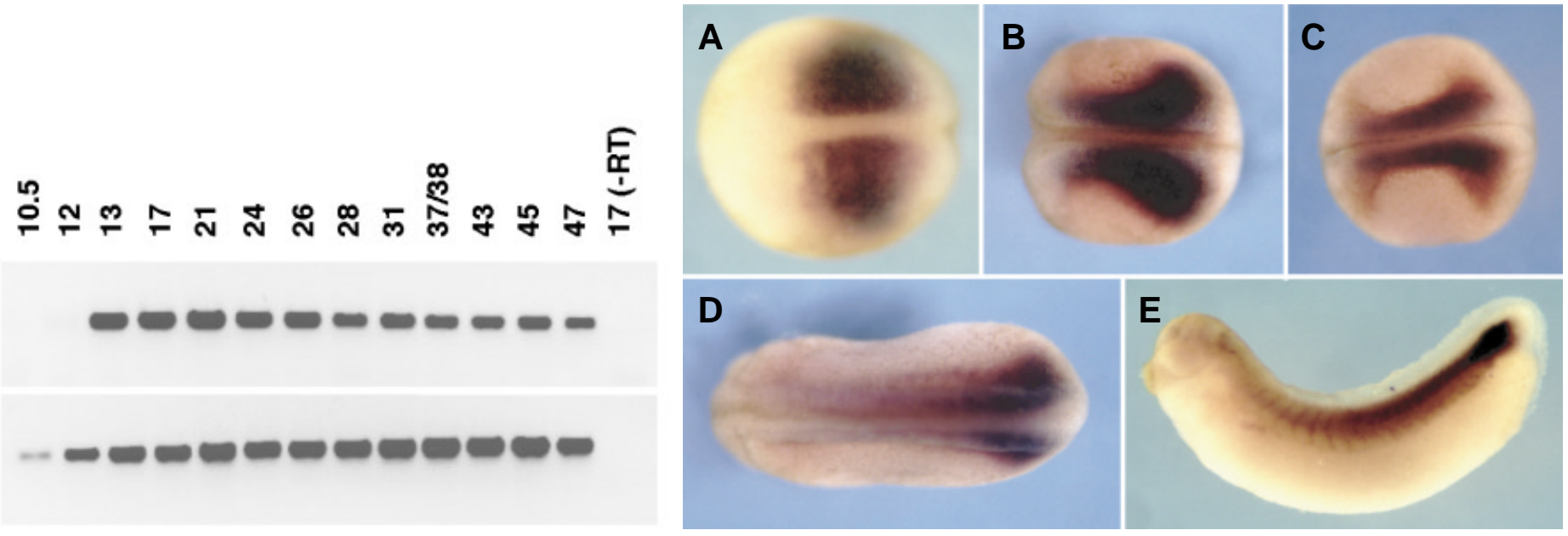

Fig. 2 (Left). Temporal expression pattern of Xenopus paraxis (top row) by RT-PCR analysis. RT-PCR was performed with total RNA from different developmental stages. The last lane was a negative control without adding reverse transcriptase. EF-1 $\alpha$ (bottom row) served as a loading control.

Fig. 3 (Right) . Whole mount in situ hybridization analysis of Xenopus paraxis mRNA expression. (A-D) Dorsal view. (E) Lateral view. All embryos are oriented with anterior to the left. (A) At stage 13, paraxis expression is in the presomitic mesoderm. (B) At stage 17, paraxis expression is reduced in the anterior region where somitogenesis begins. (C) At stage 19, paraxis becomes progressively more reduced in the anterior paraxial mesoderm. (D) At stage 24, paraxis transcripts are present at high levels only in the most caudal region where the somites are not yet compartmentalized. (E) At stage 28, paraxis expression is strong in the tip of the tail and in the dorsal half of somites from mid-trunk to hind-trunk. 


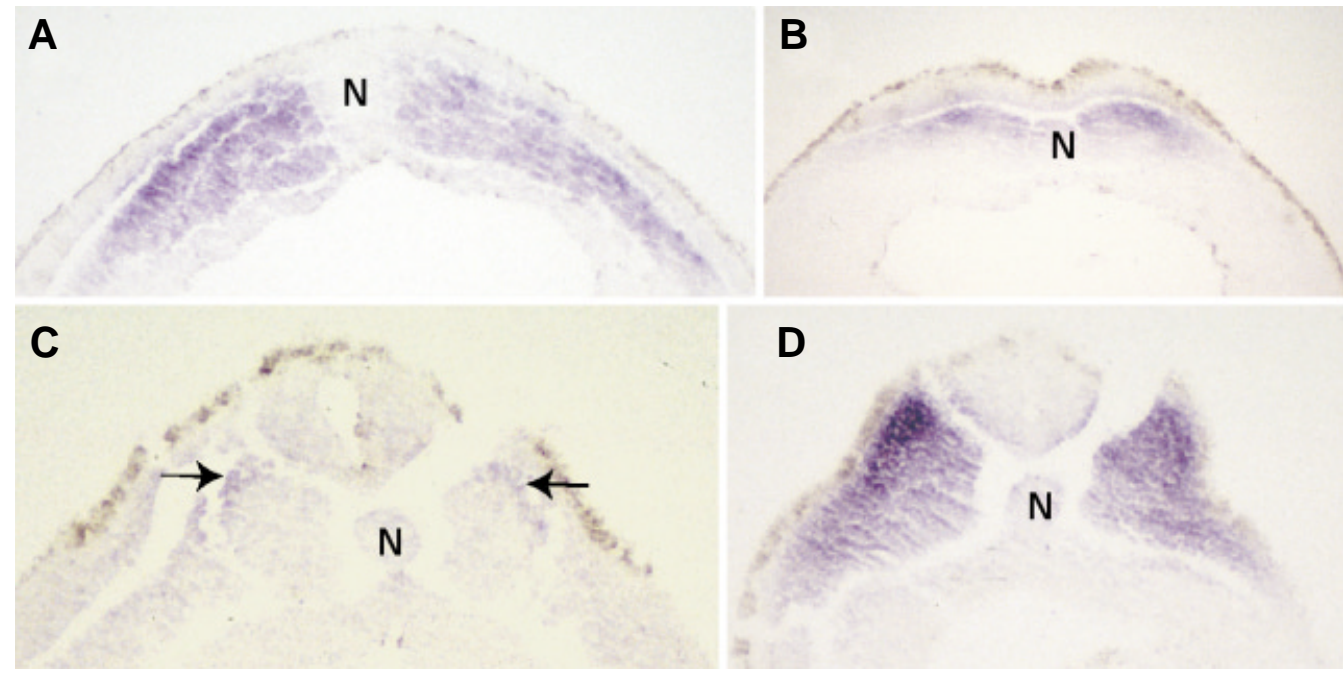

Fig. 4. Analysis of Xenopus paraxis expression in embryo sections. All sections are positioned with dorsal at the top and ventral at the bottom. (A) A stage 13 transverse section shows paraxis transcripts in the whole presomitic mesoderm, but not in the notochord. (B) A stage 17 transverse section through the anterior trunk shows expression in the dorsal region of somitic mesoderm. (C) A stage 24 transverse section through the anterior trunk shows weak expression in the dorso-lateral region of somites (arrows). (D) In contrast, a transverse section through the posterior trunk of the same embryo as in $(C)$ shows strong expression of paraxis in the entire undifferentiated somite. $N$, notochord.

\section{Spatial expression of Xenopus paraxis}

The spatial expression of paraxiswas determined by whole mount in situ hybridization. At late gastrulae, paraxis is strongly expressed in the presomitic mesoderm, but not in the notochord (Fig. 3A). As somitogenesis commences, the paraxis transcripts localize to a narrower paraxial region, lateral to the involuting neural tube (Fig. 3B). After the somites are formed, paraxis expression is progressively downregulated in the anterior paraxial mesoderm, but a high level of expression remains in the tail-bud region, where the somites are not yet differentiated (Fig. 3 C-E).

A transverse section through the trunk region of stage 13 embryo shows paraxis expression in the entire somitogenic mesoderm flanking the midline notochord (Fig. 4A). At stage 17, paraxis transcripts are preferentially localized to the dorsal portion of maturing somites (Fig. 4B). Before diminishing to undetectable levels in the rostral somites, paraxisis expressed in the most dorso-lateral region of somites, which appears to be the dermatome (Fig. 4C) (Hausen, 1991). In contrast, strong expression persists in the entire undifferentiated somites located in more caudal trunk region (Fig. 4D). Because of its expression pattern, paraxis can be used as a marker of dermatome differentiation in Xenopus. Together with the forkhead gene FoxC2 (XFKH7) (El-Hodiri et al., 2001), which is a marker of sclerotome differentiation, these two genes can be used for analysis of differentiation of the paraxial mesoderm in Xenopus.

\section{Experimental Procedures}

\section{Xenopus genomic library screening}

The full-length mouse scleraxis cDNA was used to screen the Xenopus genomic library. Positive clones were plaque purified, subcloned into pBluescript SK and sequenced.

\section{Whole mount in situ hybridization and histology}

Xenopus embryos were staged according to (Nieuwkoop, 1994). Whole mount in situ hybridizations were performed as described by (Harland, 1991). The chromogenic reaction was modified using BM purple substrate (Roche). Probes were synthesized from EcoRI linearized plasmid using T7 RNA polymerase and digoxigenin-UTP (Roche). For histological analysis, embryos were dehydrated in ethanol and embedded in paraffin after whole mount in situ hybridization. Transverse sections were cut at $12 \mu \mathrm{m}$ intervals, de-waxed in xylene and mounted with Permount (Fisher). Sections were not counterstained to retain visibility of labeled RNA transcripts.

\section{RNA isolation and RT-PCR assay}

Preparation of total RNA from animal caps using TRIzol reagent (Invitrogen) was carried out according to manufacturer's instructions. RT-PCR was performed by using the following primers and cycling conditions. For: paraxis (55드, 30 cycles; forward, 5'-GCG TAA ACA CCG CTT TCA CCG; reverse, 5'-CCT TGG CTC TGT AGA CGG TAC). For EF1alpha that was used as a positive control: (55으, 26 cycles; U: 5'-CAG ATT GGT GCT GGA TAT GC; R: 5'-ACT GCC TTG ATG ACT CCT AG).

\section{Acknowledgements}

We thank Dr. E. Olson for providing mousescleraxis probe, C. Zilinski for a critical reading of this manuscript and Dr. H. El-Hodiri, Dr. L. Zhang, Dr. O. Medina-Martinez and Dr. I. Brownell for technical guidance.

\section{References}

BARNES, G.L., ALEXANDER, P.G., HSU, C.W., MARIANI, B.D. and TUAN, R.S (1997). Cloning and characterization of chicken paraxis: A regulator of paraxial mesoderm development and somite formation. Dev Bio/189: 95-111.

BROWN, D., WAGNER, D., LI, X., RICHARDSON, J.A. and OLSON, E.N. (1999). Dual role of the basic helix-loop-helix transcription factor scleraxis in mesoderm formation and chondrogenesis during mouse embryogenesis. Development 126: 4317-29.

BURGESS, R., CSERJESI, P., LIGON, K.L. and OLSON, E.N. (1995). Paraxis: A basic helix-loop-helix protein expressed in paraxial mesoderm and developing somites. Dev Bio/168: 296-306.

BURGESS, R., RAWLS, A., BROWN, D., BRADLEY, A. and OLSON, E.N. (1996). Requirement of the paraxis gene for somite formation and musculoskeletal patterning. Nature 384: $570-3$.

CSERJESI, P., BROWN, D., LIGON, K.L., LYONS, G.E., COPELAND, N.G., GILBERT, D.J., JENKINS, N.A. and OLSON, E.N. (1995). Scleraxis: A basic helix-loop-helix protein that prefigures skeletal formation during mouse embryogenesis. Development 121: 1099-110. 
EL-HODIRI, H., BHATIA-DEY, N., KENYON, K., AULT, K., DIRKSEN, M. and JAMRICH, M. (2001). Fox (forkhead) genes are involved in the dorso-ventral patterning of the Xenopus mesoderm. Int J Dev Bio/45: 265-71.

HAMILTON, L. (1969). The formation of somites in Xenopus. J Embryol Exp Morphol22: 253-64.

HARLAND, R.M. (1991). In situ hybridization: An improved whole-mount method for Xenopus embryos. Methods Cell Bio/36: 685-95.

HAUSEN, P., M. RIEBESELL. (1991). The early development of Xenopus laevis: An atlas of the histology. Springer-Verlag, Berlin.

HEIN, J. (1990). Unified approach to alignment and phylogenies. Methods Enzymol. 183: $626-45$

KELLER, R. (2000). The origin and morphogenesis of amphibian somites. Curr Top Dev Biol 47: 183-246.

MORIN-KENSICKI, E.M. and EISEN, J.S. (1997). Sclerotome development and peripheral nervous system segmentation in embryonic zebrafish. Development 124: 159-67.

NIEUWKOOP, P.D., J. FABER. (1994). Normal table of Xenopus laevis (daudin). Garland Publishing. Inc., New York and London.
QUERTERMOUS, E.E., HIDAI, H., BLANAR, M.A. and QUERTERMOUS, T. (1994). Cloning and characterization of a basic helix-loop-helix protein expressed in early mesoderm and the developing somites. Proc Nat/ Acad Sci USA 91: 7066-70.

SCHWEITZER, R., CHYUNG, J.H., MURTAUGH, L.C., BRENT, A.E., ROSEN, V OLSON, E.N., LASSAR, A. and TABIN, C.J. (2001). Analysis of the tendon cell fate using scleraxis, a specific marker for tendons and ligaments. Development 128: 3855-66.

SHANMUGALINGAM, S. and WILSON, S.W. (1998). Isolation, expression and regulation of a zebrafish paraxis homologue. Mech Dev 78: 85-9.

YOUN, B.W. and MALACINSKI, G.M. (1981). Somitogenesis in the amphibian Xenopus laevis: Scanning electron microscopic analysis of intrasomitic cellular arrangements during somite rotation. J Embryol Exp Morpho/64: 23-43.

Received: September 2004 Reviewed by Referees: September 2004 Modified by Authors and Accepted for Publication: September 2004 Edited by: Thomas D. Sargent 